B. Gallardo-Paúls

V. Moreno-Campos

\title{
Evolución de la pragmática en un caso de afasia de Broca severa
}

Departamento Teoria dels Llenguatges

Universitat de Valéncia-Estudi General València

\section{Resumen}

Aunque es ya frecuente hablar de la pragmática de los pacientes con daño cerebral lo cierto es que son bien pocos los análisis detallados y rigurosos de la producción lingüistica real de tales pacientes. En este trabajo presentamos un modelo de análisis pragmático a partir de tres grabaciones realizadas a un pacientes con afasia motora. Analizamos la evolución pragmática mediante el registro cuantitativo de datos referidos al indice de participación conversacional, al tipo de intervenciones, y al tipo de actos de habla. Se identifican como categorias especificas del habla afásica las "tareas de edición»: actos borrador, actos proposicionales y actos activadores de inferencia (interjecciones y actos locutivos), que rentabilizan otras categorias enunciativas como las implicaturas y el significado inferencial. Los datos pertenecen al corpus PerLA (percepción, lenguaje y afasia), integrado por grabaciones conversacionales realizadas a pacientes afásicos. Nuestro análisis de la evolución del paciente afásico evidencia un aumento claro del dominio léxico y gramatical, junto a una reducción llamativa de su índice de participación conversacional. De ahi la necesidad de que la rehabilitación logopédica habitual, preocupada por la recuperación de la gramática, deba incorporar una consideración real y efectiva de los elementos pragmáticos. Para ello es necesario que la evaluación pragmática sea rigurosa y detallada.

Palabras dave: Actos de habla, afasia, conversación, daño cerebral, datos etnográficos, evaluación, lingüistica interaccional, pragmática

\section{Pragmatic evolution in a case of severe Broca's aphasia}

Though it is usual to speak about pragmatics of the speakers with brain damage, there are few detailed and rigorous analyses of the linguistic real production of such speakers. In this paper we present a model of pragmatic analysis from several transcriptions of a speaker with Broca aphasia by registered quantitatively information recounted to the index of conversational participation, to the type of interventions, and to the type of speech acts. There are identified as specific categories of the aphasic speech the editing tasks: draft acts, propositional acts, and activators of inferences acts (interjections and locutive acts), that overexploit other pragmatics cathegories as the Grice's implicatures or the inferencial meaning. The data belong to the corpus PerLA (perception, language and aphasia), integrated by conversational recordings of aphasic speakers. The evolution of the aphasic speaker demonstrates a clear increase of the lexical and grammatical skills, close to a showy reduction of conversational participation index. Usual speech therapy, worried by the recovery of the grammar, has to incorporate a real and effective consideration of the linguistic pragmatic elements, so that the lexical and morphosyntactic rehabilitation is not realized with the lost of the

Esta investigación se realiza en el marco de los proyectos del Ministerio de Educación y Ciencia, «ariables pragmáticas en la evaluación y rehabilitación de afasiasn (Ref. HUM2004-05847-C0202/FILO) y "Elaboración y análisis pragmático de un corpus de lenguaje afásicon (Ref. BFF2002-00349), ambos con dotación adicional de los Fondos FEDER y el segundo de ellos también con una ayuda complementaria de la Generalitat Valenciana (GRUPS2004/14) 
communicative efficiency of the speaker. For that, it will be necessary that the pragmatic evaluation be rigorous and detailed.

Key Words: Aphasia, assessment, brain damage, conversation, ethnogra phical data, pragmatics, speech ácts

\section{Introducción}

Desde los trabajos pioneros de Penn (1985) o Prutting y Kirchner $(1983,1987)$, las propuestas de evaluación de la pragmática en pacientes con patologias de incidencia verbal se pueden agrupar en dos categorias: las que utilizan técnicas desencadenantes planificadas (Creaghead, 1984; Schulman, 1985; Gardner y Brownell, 1986; Bryan, 1989; Phelps-Terasaki y Phelps-Gun, 1992; Linscott, Knight y Godfrey, 1996; Puyuelo, Wiig, Renom y Solanas, 1997; Kasher, Batori, Soroker, Graves y Zaidel, 1999), y las que se elaboran a partir de datos obtenidos con validez ecológica (Gerber y Gurland, 1989; Terrell y Ripich, 1989).

Entre las primeras, la evaluación de la pragmática suele ser parcial, referida básicamente a categorías enunciativas como los actos de habla y las implicaturas griceanas; asi ocurre, por ejemplo, con la Batería de Comunicación del Hemisferio Derecho (Gardner y Brownell, 1986), la BLOC (Puyuelo, Wiig, Renom y Solanas, 1997) o la Batería Pragmática (Kasher, Batori, Soroker, Graves y Zaidel, 1999), cuya base epistemológica de referencia está en las teorías enunciativas clásicas de Austin, Searle o Grice. Por el contrario, las pruebas de evaluación que se basan en datos obtenidos etnográficamente, como el Protocolo Pragmático (Prutting y Kirchner, 1983) o el Perfil de Adecuación Comunicativa (Penn, 1985) incluyen visiones más amplias de componente pragmático, incorporando la evaluación de elementos propios de la pragmática textual (destacan en este sentido el Perfil de Habilidades Discursivas de Terrell y Ripich, 1989, el PELC-HD de Labos y cols., 2000 o el MTBA de Labos y cols., 2005 por su atención a las superestructuras) y de la pragmática interaccional (ámbito en el que destacamos el ALICC de Adams y Bishop, 1989; los Perfiles de Análisis Conversacional de Witworth, Perkins y Lesser, 1997 y el protocolo de Cuantificación de Conductas Conversacionales de Crockford y Lesser, 1994).

Es bien sabido que, a diferencia de lo que ocurre en los componentes fonológicos, morfosintácticos o semánticos, la evaluación pragmática no puede realizarse exclusivamente mediante tests al uso, ya que tales pruebas no incorporan debidamente las condiciones enunciativas (Muñoz-Céspedes y Melle, 1999; Penn, 2000; Adams, 2000; Baixauli-Fortea, Roselló y Miranda, 2004); esto es debido a que la pragmática sólo puede valorarse desde el propio contexto de uso verbal, y no se define por referencia a valores de corrección, sino de efectividad comunicativa. La utilidad de las pruebas basadas en técnicas desencadenantes ha de ser complementaria, y estriba en su valor para provocar la ocurrencia de conductas y categorías que no hayan aparecido en los datos naturales, aunque esta capacidad desencadenante no es fácil de garantizar. Por lo demás, resulta del todo inadecuado tratar de evaluar exclusivamente el uso pragmático de un hablante proponiéndole ítems desencadenantes para los que establezcamos previamente reacciones correctas (tachando además de incorrectas las que se alejen de nuestra idea).

Estas circunstancias explican que la manera habitual de proceder consista en realizar grabaciones conversacionales que se transcriben y se analizan a posteriori. Obviamente, es un tipo de análisis que exige más tiempo que la aplicación de un test (especialmente por lo ingrato de la labor transcriptora), pero que resulta necesario si de verdad queremos describir pragmáticamente la producción verbal de un paciente. La critica más repetida a este tipo de evaluación se refiere, evidentemente, a las dificultades para objetivar y jerarquizar resultados que se describen como "adecuados", "básicamente adecuados", "inadecuados», etc. Nuestra intención en el presente trabajo es presentar un modelo que trata de conjugar las bases teóricas de la pragmática con ese intento cuantificador $y$, por tanto, objetivable.

Desde el comienzo de la elaboración del corpus PerLA dedicado al lenguaje afásico (Gallardo, 2002; Gallardo y Sanmartín, 2005; Gallardo y Moreno, 2005a) hemos tenido presentes algunas asunciones fundamentales como las siguientes:

- Necesidad de datos con validez ecológica (Joanette y Ansaldo, 1999; Perkins, Withworth y Lesser, 1998; Vanhalle, Lemieux, Ska y Joanette, 2000) y, por tanto, un método etnográfico.

- Participación de los interlocutores-clave en las grabaciones.

- Transcripción etnometodológica (Jefferson, 1992).

- Consideración de variables pragmáticas.

Una vez validadas las transcripciones iniciales, tras los necesarios filtrados por parte de varios 
investigadores, empieza el análisis propiamente dicho, para lo que hemos constituido una base de datos que se va incrementando a medida que nuestro interés incluye diferentes categorias y nuevas transcripciones. Dedicamos el presente trabajo a uno de tales análisis, que selecciona en concreto tres grabaciones del PerLA pertenecientes a un mismo paciente, el Sr. ARC, remitido por el Servicio de Neurología del Hospital Clínico de Valencia tras haber sufrido un accidente cerebrovascular (ACV) el 23 de noviembre de 2003. Nuestro objetivo fundamental en este artículo es presentar las fases básicas del análisis de los datos, como paso previo que pueda concretarse en futuros protocolos de evaluación y rehabilitación pragmática.

\section{Caso ARC: las tres grabaciones}

\section{Datos}

Las grabaciones analizadas para el presente trabajo proceden, como hemos adelantado, del corpus PerLA, que se viene eläborando desde el año 2000 en el área de lingüistica general de la Universitat de ValènciaEstudi General, gracias a convenios de colaboración firmados con el Servicio de Neurologia del Hospital Clinico de Valencia, el Instituto de Neuro-reabilitación y Afasia INIA-NEURAL, y el Servicio de Daño Cerebral de Hospitales NISA. Hasta el momento, el corpus consta de 7 informantes con afasias mixtas, 15 de afasia no fluente y 5 de afasia fluente; en el último año hemos ampliado el tipo de datos incluyendo también otras situaciones en las que sean previsibles alteraciones pragmáticas, y que puedan por tanto servir como corpus de contraste; así, hemos incorporado grabaciones pertenecientes a 7 pacientes con traumatismo cráneoencefálico que no han desarrollado afasia, 4 lesionados en hemisferio derecho, hablantes con TDAH y 6 con síndrome de Williams; las grabaciones continúan.

ARC tiene 58 años en el momento del ACV y es diestro; sus datos biográficos presentan a un hombre poliglota, con el gallego como lengua materna (aunque sólo utilizada en el entorno familiar de la infancia y primera juventud), y el español como lengua habitual en sus relaciones familiares y sociales; 12 años de emigrante en Alemania y dos en Australia le han llevado a cierto dominio del alemán, inglés e italiano oral; sólo del alemán tenía cierto dominio escrito; regresó a España y se instaló en Valencia hace ya 32 años, durante los cuales el español ha sido su lengua habitual.
El informe médico de la resonancia magnética (RM) del 4 de diciembre de 2004 muestra "una extensa lesión isquémica en estadio agudo/subagudo localizada en el hemisferio cerebral izquierdo, con afectación fronto-temporal-parietal y un mínimo componente hemorrágico petequial»; el diagnóstico inicial de afasia global tiene un tránsito rápido a afasia de Broca severa, en que la comprensión se conserva pese al altísimo déficit expresivo. Disponemos de datos de dos evaluaciones logopédicas, realizadas en enero de 2004 y en febrero de 2006, y cuyos resultados consignamos en el anexo 1.

Grabación ARC1. En esta grabación, 6 semanas después del ACV, participan tres interlocutores-clave, además del informante y la entrevistadora (hija, esposa y nieto, aunque éste apenas interviene). Junto al déficit estrictamente verbal, el hablante presenta hemiplejia derecha, lo que limita también su movilidad y su lenguaje no verbal. La grabación dura 42: $44 \mathrm{~min}$.

Grabación ARC2. Esta grabación tiene lugar 12 semanas después del $A C V$; participan sólo la investigadora, el informante y su esposa. Dura 31: $55 \mathrm{~min}$.

Grabación ARC3. Esta nueva grabación tiene lugar en abril de 2005, por lo que han transcurrido casi 16 meses desde el ACV; su duración es de 33: $38 \mathrm{~min}$. Participan, como en ARC2, el hablante afásico, su esposa y la entrevistadora.

Las tres grabaciones fueron transcritas a partir de las convenciones etnometodológicas desarrolladas por Jefferson tal y como han sido adaptadas por el grupo PerLA (Gallardo y Sanmartín, 2004); las dos primeras han sido publicadas en el segundo volumen del corpus, dedicado a afasia no fluente. La metodologia coincide con el método sociolingúistico de observación participante, algo que minimiza las consecuencias de la paradoja laboviana del observador (Labov, 1972), a pesar de que, obviamente, los participantes son conscientes de estar siendo grabados y asumen como objetivo de tal grabación la participación del hablante afásico. La necesidad de incluir en la grabación a los interlocutores-clave (concepto que hemos tomado de Witworth, Perkins y Lesser en su Perfil de Análisis Conversacional para Personas con Afasia) se relaciona precisamente con la búsqueda de datos que se aproximen todo lo posible al uso real del lenguaje.

\section{Elementos analizados en las tres grabaciones}

A continuación presentamos los elementos pragmáticos cuyo análisis nos parece relevante en la 
caracterización lingüistica de cada informante con afasia. Como puede verse, nuestra propuesta atiende a las categorias básicas utilizadas por la pragmática, $y$ trata de aportar una perspectiva cuantificadora necesaria para objetivar la evaluación.

\section{El índice de participación conversacional (IPC)}

Es el primer elemento que consideramos en cada transcripción, utilizado también como dato inicial por Adams y Bishop en su descripción del desorden semántico-pragmático (Adams y Bishop, 1989) y por Crockford y Lesser en su protocolo CCC (Cuantificación de Conductas Conversacionales, Crockford y Lesser, 1994). El IPC no se expresa en datos absolutos, ya que cada grabación tiene distintos interlocutores y duración, sino relativos: se trata del porcentaje de intervenciones o palabras emitidos por el hablante. Para el IPC de intervenciones contabilizamos los turnos de cada participante, incluyendo indistintamente los que constituyen verdaderas intervenciones y los que pertenecen al sistema secundario, es decir turnos de oyentes o continuadores, a veces llarliados feedback o retroalimentación pese a que mantienen estática la distribución del turno: sí, ya, claro, ajá,... y convencionalmente uh um. Esta equiparación se debe a que en los casos severos resulta muy dificil delimitar las intervenciones reactivas evaluativas y estas emisiones tipicas de la posición del oyente (una diferencia, p. ej., respecto al análisis de Adams y Bishop del TSP, donde la fluidez verbal no está afectada como en la afasia; ambas autoras contabilizan las "respuestas minimas" como una categoría específica).

EI IPC informa sobre la voluntad comunicativa del hablante, así que tiene un interés psicológico y se relaciona también con el carácter individual de cada uno y su actitud frente a la afasia. Puesto que el objetivo que todos los participantes asumen para cada grabación es lograr datos hablados del participante afásico, lo esperable en nuestro corpus es que sea tal hablante quien protagonice la transcripción. Como ocurre en casi todas las categorias pragmáticas, esta cifra (ya sea en números absolutos o en porcentaje respecto a la duración total de la conversación) no tiene un referente de corrección o norma (Moreno, 2006. Se ha comparado el IPC de los datos PerLA con un corpus de interacciones coloquiales); en cualquier interacción encontramos hablantes con distinto nivel de participación, y un mismo interlocutor puede presentar variaciones notables respecto a cuánto habla según cuándo, de qué y con quién (las clásicas varia- bles sociolingüisticas). Pero lo más notable en el corpus son los casos de absoluto desequilibrio entre los participantes, desequilibrios que vinculamos no sólo a la inhibición o desinterés del paciente afásico, sino también a la participación a veces desmesurada de su interlocutor-clave; en este sentido cabe señalar que la elaboración de cursos y quias de habilidades comunicativas destinados específicamente a estos pacientes que se relacionan con el afásico (Gallardo, 2005a) contribuyen (entre otras cosas) a concienciarlos de la necesidad de dejar libre el turno de palabra con un tempo distinto al habitual antes de la afasia.

\section{Intervenciones}

El primer paso en el análisis de la grabación transcrita es la clasificación de cada intervención según una tipología pragmática, a fin de obtener (también en términos de porcentaje) el predominio de uno u otro tipo en cada paciente. Al plantearnos tanto la ilocutividad como la orientación interactiva de las intervenciones pronunciadas por los pacientes afásicos en entornos conversacionales, nos encontramos intervenciones en las que no es posible identificar estructuras sintácticosemánticas completas, sino elementos aparentemente inconexos y carentes de significado, cuya interpretación se basa completamente en el contexto y la comunicación no verbal simultánea. Este tipo de turnos aparecen a veces en la interacción cotidiana, pero no con el nivel de frecuencia de la conversación afásica; nos referimos a intervenciones aparentemente "vacias", que sin embargo realizan un innegable papel interactivo; he aquí algunos ejemplos:

0545 l: ¿eeh?/ puees $\rightarrow / /$ (MOVIMIENTOS RÁPIDOS DE LA MANO ABIERTA) perOo $\rightarrow 100 \rightarrow(\Rightarrow M$, DETIENE LA MANO APIÑANDO LOS DEDOS, MIRA HACIA DELANTE, CIERRA LOS OJOS) jaay!// (2.0, SE RECUESTA, $\Rightarrow M)$ uum $\rightarrow$

0652 ।: eso $\uparrow$ ay $\uparrow$ ay $\uparrow$ ay $\uparrow /$ tot - jay! $/(\Rightarrow E$, compÁs indICE) mira yo $\uparrow$ estoy/ en- en que llegueaah- (MOVIMIENTO CIRCULAR CON LA MANO) ya $\uparrow$ vamos $\rightarrow$

¿Cómo caracterizar estas intervenciones con una estructura sintáctica ciertamente alterada, tan rica en elipsis, anacolutos y formas quebradas o interrumpidas, pero que sin embargo consiguen cumplir su papel como intervenciones en el desarrollo lineal de una toma de turno? El estudio de este tipo de intervenciones, asi como su frecuencia de aparición, nos llevó a la conclusión de que es posible 
adscribirles una función interactiva real, coherente con los modelos de análisis pragmático desarrollados para la conversación natural no marcada. Asumimos así una de las premisas fundamentales propuestas por Harvey. Sacks para el análisis conversacional etnometodológico, según la cual el enfoque del analista ha de incorporar necesariamente la interpretación que los propios participantes hacen del acto conversacional; de este modo, si el interlocutor-clave da por válida una intervención como las que acabamos de transcribir, nuestro análisis les concederá también un papel interactivo. En definitiva, las categorías utilizadas en este análisis de intervenciones son las habituales en pragmática interactiva (Gallardo, 1993):

- Inicio: turno de habla con orientación interactiva desencadenante, predictiva, que impone al interlocutor restricciones conversacionales (básicamente de sintaxis interrogativa, aunque también se incluyen aquí los juicios que se interpretan como petición de conformidad/disconformidad) [1].

- Respuesta: turno de habla que ha sido predicho por el anterior [R]; junto a las respuestas correctas o pragníáticamente adecuadas [R], marcamos especificamente las ocasiones en que esta respuesta es ecoica, y repite total o parcialmente la intervención predictiva $[R=]$; las respuestas que evidencian una mala interpretación o comprensión del turno predictivo anterior se registran como [R-].

- Informe: intervenciones con carga semántica que hacen progresar la información, aunque (a diferencia de lo que ocurre en el análisis de la conversación no afásica) en gran número de casos no se trata de intervenciones con sentido completo [Inf]; son iniciales pero no predictivas.

- Reacción evaluativa: turno de habla reactivo, pero no predictivo ni predicho, próximo al continuador [R.Ev.].

En el análisis de los datos resulta muy significativo que cierto hablante emita turnos básicamente reactivos, o que por el contrario demuestre su intención comunicativa recurriendo a turnos inciativos, como las preguntas o los informes.

\section{Actos de habla}

El paso siguiente, una vez identificado el tipo de intervenciones, es el análisis concreto de los actos de habla que las rellenan. Aquí, junto a la cla- sificación pragmática habitual teniendo en cuenta la ilocutividad y la orientación interactiva, necesitamos registrar algunas modalidades especificas de actos que se pueden explicar por compensación del déficit gramatical; con la utilización de este tipo de actos, el hablante afásico logra una comunicación que no se basa en la semanticidad de sus intervenciones (Grice, 1957, lo identificaba como significado explicito), sino en la suma de códigos verbales y paraverbales, junto al recurso a la capacidad inferencial del interlocutor. Asi, ante la limitación para construir enunciados, el hablante utiliza todos sus recursos para convertir la enunciación en enunciado, trasladando al otro interlocutor el peso de la comunicación explícita.

A diferencia de lo que ocurre en el ámbito de las intervenciones, en el análisis concreto de los actos de habla si introducimos algunos tipos que resultan especialmente rentables en el habla de los participantes afásicos. De hecho, la separación de niveles entre intervenciones y actos nos permite reflejar una peculiaridad del habla afásica: la disociación entre las distintas dimensiones de los actos de habla identificadas por la pragmática enunciativa clásica (Austin, 1962; Searle, 1976). Mientras asignamos a las intervenciones la dimensión ilocutiva básica que refleja la intención del hablante (básicamente intervenciones inicitivas y reactivas), al caracterizar los actos verbales damos cuenta separadamente de la doble dimensión que propone Searle para el acto locutivo austiniano:

- Una dimensión estrictamente enunciativa o locutiva (un soporte verbal que transporta el acto ilocutivo sin llegar a darle carga semántica).

- Una dimensión proposicional (dotada de un claro valor referencial).

De este modo, los actos de habla manejados en el análisis del corpus a tenor de estas matizaciones pragmáticas son los siguientes (con un grado decreciente de semanticidad):

- Actos proposicionales [AP]: los que poseen una carga semántica basada en el léxico de la lengua y, por tanto, hacen progresar informativamente la conversación; por supuesto, este uso léxico puede ser deficitario, con sintomas como agramatismo o paragramatismo, pero responde al uso habitual de las lenguas naturales. Este tipo de acto de habla supone a veces un contenido semántico mínimo, lo que nos aproxima a la categoría que Tomoeda y Bayles (1993) identifican como unidades de 
información" para el habla de personas con Alzheimér.

- Actos activadores de inferencias: este tipo de actos de habla es especialmente frecuente en las afasias motoras, pues el hablante los utiliza para explotar la capacidad inferencial de su interlocutor y lograr una construcción colaborativa de la interacción; con frecuencia son monolexemáticos, presentan alargamientos y entonación suspendida, y suelen acompańarse de ilustradores o reguladores. Estos son:

- Interjección [Int.]: se trata de exclamaciones que pueden tener un contenido semántico de lexicalización variable; como sabemos, la interjección se caracteriza pragmáticamente por su "ausencia de valor conceptual" (López Garcià, 1989) y por la total absorción del enunciado por la enunciación. En este sentido, la interjección emerge como una categoría morfosintáctica comodín, capaz de adoptar cualquier valor proposicional que le asigne la interpretación contextual; esta flexibilidad la convierte en una proforma por excelencia, ya que cualquier elemento de la lengua puede servir de soporte para la interjecciól;; de ahí que pueda caracterizarse también como unidad léxica no descriptiva dotada de función conectora (Vázquez, 2003).

- Actos locutivos [AL]: son emisiones de relleno muy próximas a las pausas oralizadas, con las que el hablante materializa su turno sin decir realmente nada en términos de gramaticalidad; estos actos pueden entenderse como una separación de las dimensiones locutiva y proposicional del acto de habla, de tal manera que hay locución pero a partir de ella no se puede extraer un contenido semántico o informativo real; es frecuente la utilización de marcadores discursivos y pronombres con esta función, asi como una pronunciación alargada (yooo..., éeel..., pueees...).

- Tareas de edición: aceptamos la propuesta de Crockford y Lesser en su protocolo de Cuantificación de Conductas Conversacionales, aunque con ligeras variaciones. En otras ocasiones hemos descrito estas conductas equiparándolas a las tareas de montaje cinematográfico, en las que el montador desecha las tomas falsas y monta las que configuran la edición final. Existen varias conductas con esta función:

- Pausas oralizadas [P0]: cuantificamos las vocalizaciones con las que el hablante rellena el silencio para no perder el turno de palabra mientras planifica su intervención (Jaffe $y$
Feldstein, 1970); nuestras convenciones de transcripción utilizan la convención estándar de marcar estas prolongaciones paralingüisticas con un máximo de tres vocales (uuum, eeeh), asi que no tenemos en cuenta la duración real de tal vocalización.

- Pausas vacías de al menos dos segundos de duración [PV]; los etnometodólogos identificaron como duración estándar de la pausa interior de turno las de 1 s (Jefferson, 1989). Crockford y Lesser proponen incluir entre las tareas de edición las pausas superiores a $2 \mathrm{~s}$, y este es el criterio que hemos adoptado.

- Actos borrador $[A B]$ : incluimos aquí las expresiones que emite el paciente en su esfuerzo por recorrer cierta serie léxica hasta encontrar el elemento léxico que desea, así como los intentos fallidos de encontrar cierta palabra o expresión, los circunloquios que evidencian problemas de acceso léxico, o las preguntas específicas sobre denominación.

- Preguntas confirmatorias (tag question) [PC]: las incluimos entre las tareas de edición porque son un instrumento que utiliza el hablante afásico para verificar la comprensión por parte de su interlocutor, en la medida en que le proporciona un posible lugar de interrupción (lugar de transición pertinente, LTP). De ahí que encontremos casos de afasia motora con una sobreexplotación de este tipo de preguntas: ¿no?, ¿eh? En la conversación no afásica (Dubois y Crouch, 1975; Davidson, 1984) las PC ocupan tipicamente la posición de enlace con intervenciones posteriores (posición 3 en la estructura ideal de la intervención); sin embargo, en las conversaciones afásicas estas emisiones interrogativas no suponen una cesión real del turno conversacional (lo que supondría la activación de la primera regla de cambio de hablante propuesta por Sacks, Schegloff y Jefferson: selección directa), sino que por el contrario se utilizan como estrategia de mantenimiento del turno.

- Actos no verbales: la transcripción intenta reflejar los gestos utilizados por el hablante en tres niveles básicos: los emblemas, los ilustradores y los reguladores, adoptando la clasificación básica de conductas no verbales que propusieron Eckman y Friesen (1969). Especialmente en los pacientes con afasias motoras esta información resulta imprescindible, pues el gesto es con bastante frecuencia acompañante y matizador del habla, y llega en ocasiones a sustituirla por completo. Codificamos [NV] los actos no verbales considerados pertinentes por la transcripción. 
Longitud media de turno

Es decir, el número de unidades totales contabilizadas en la participación del informante, dividido entre el número de turnos que emite en la interacción. Esta proporción se calcula de dos maneras:

- En actos de habla, a partir de la clasificación expuesta en el apartado anterior.

- En número de palabras, a partir del cómputo que realiza el procesador de textos, que identifica palabras y seudopalabras.

La longitud media de turno (unidad natural, Allerton, 1969) es la adaptación pragmática de una medida habitual en afasiologia, como es la longitud media de frase (unidad gramatical). Este medida nos orienta sobre la fluidez del hablante afásico, y cuando la aplicamos a todos los implicados en la conversación nos habla de predominios discursivos, por lo que se trata de un instrumento pertinente también al caracterizar la conducta de los interlocutores-clave.

\section{$i^{2}$ \\ Pragmática téxtual}

En esta fase analizamos los aspectos gramaticales que puedan ser de relevancia, y que completan cualitativamente el análisis cuantitativo. Uno de los elementos más significativos respecto a la compensación pragmática del déficit gramatical es el uso enfático de elementos de coherencia y conexidad, como los marcadores discursivos (Gallardo y Marín 2005), que rellenan frecuentemente actos activadores de inferencias; estos elementos configuran la dimensión "arquitectónica" del discurso, y se ha comprobado su pérdida en las alteraciones verbales ocasionadas por demencia (Ripich, Carpenter y Silo, 2000; Dijkstra, Bourgeois, Allen y Burgio, 2004). Otro aspecto importante se refiere a los agramatismos y paragramatismos, estos más relacionados con la cohesión de estructura superficial. Aunque no es un tipo de análisis pertinente en casos de hablantes con afasia severa, también situamos en este nivel los aspectos referidos al significado inferencial, es decir, presuposiciones, inferencias trópicas lexicalizadas, y actos de habla indirectos que suponen la transgresión de alguna máxima conversacional. Aunque las presuposiciones no son habitualmente consideradas por la lingüistica clinica, si existe ya cierta tradición en el estudio de los modismos y unidades fraseológicas, que responden al componente semántico de cada gramática (Kerbel y Grunwell, 1998; Nenonen, Niemi y Laine, 2002), y de las inferencias trópicas no lexicalizadas, como metáforas, ironias y sarcasmos, que se derivan generalmente de la indirección y transgresión de implicaturas (Brownell y Stringfellow, 1999; Tartter, Gomes, Dubrovsky, Molholm y Stewart, 2002; Champagne, Desautels y Joanette, 2003; Schmidt, DeBuse y Seger, 2005). Se trata de categorias que habitualmente son tenidas en cuenta por las pruebas de evaluación específicamente diseñadas para el hemisferio derecho, como las de Gardner y Brownell, o la de Bryan.

En los hablantes que tienen preservada una minima fluidez verbal resulta también interesante el análisis de las superestructuras textuales narrativas y argumentativas (según Bruner (1993) los moldes textuales básicos), por lo general preservadas en la afasia. Aunque la bibliografía ofrece algunos estudios relativos a la narración en la afasia (Davis y Coelho, 2004; Marini, Carlomagno, Caltagirone y Nocentini, 2005), sólo en contadas ocasiones se trabaja con el concepto de superestructura; una excepción notable la constituye el protocolo para hablantes con lesión en el hemisferio derecho de Labos, Zabala, Atlasovich, Pavón y Ferreriro (2003), y el trabajo de Hamel, Giroux y Joanette (2003) sobre la superestructura argumentativa del silogismo en lesionados de hemisferio derecho.

\section{Resultados}

En los siguientes apartados mostramos el resultado de aplicar el modelo de análisis descrito a tres grabaciones de un mismo paciente con afasia.

\section{El indice de participación conversacional (IPC)}

En el contraste con otras transcripciones del PerLA llama la atención el alto índice de participación conversacional mostrado por $A R C$, especialmente en las grabaciones iniciales (ARC1 y ARC2), en que su capacidad de emitir palabras (y por tanto, actos proposicionales) es casi nula.

Los gráficos reflejan para las tres grabaciones la participación conversacional del Interlocutor Afásico (IA), los Interlocutores-Clave (IC-1, la esposa en los tres casos, e IC-2, la hija en ARC1) y la entrevistadora (E). La figura 1 muestra la emisión de palabras/seudopalabras y la figura 2 refleja el cómputo de turnos. 


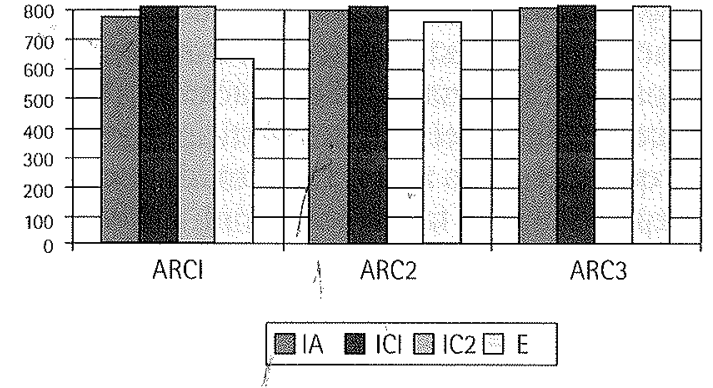

Figura 1 Indice de participación conversacional en las tres grabaciones (palabras).

\section{Intervenciones}

Atendemos en primer lugar a clasificar las intervenciones emitidas por el hablante afásico según su orientación interactiva; este dato nos parece altamente significativo (Gallardo y Hernández, 2006; Gallardo y Moreno, 2006), puesto que la emisión de turnos iniciales, no predichos por ningún otro participante, evidencia lógicamente una iniciativa comunicativa y participativa mayor que los turnos de respuesta, obligados por intervenciones ajenas; como hemos adelantado anteriormente consideramos que las intervenciones pueden clasificarse como inicios, respuestas, informes, y reacciones evaluativas.

En el análisis secuencial de las tres grabaciones, la información más relevante en la distribución de intervenciones es la diferencia en intervenciones predichas, es decir, "arrastradas" por la orientación interactiva del interlocutor (inicios, informes y reacciones evaluativas).

La tabla 1 muestra los tipos de intervención utilizados por ARC en cada grabación, calculando para cada valor absoluto el valor porcentual respecto al total de

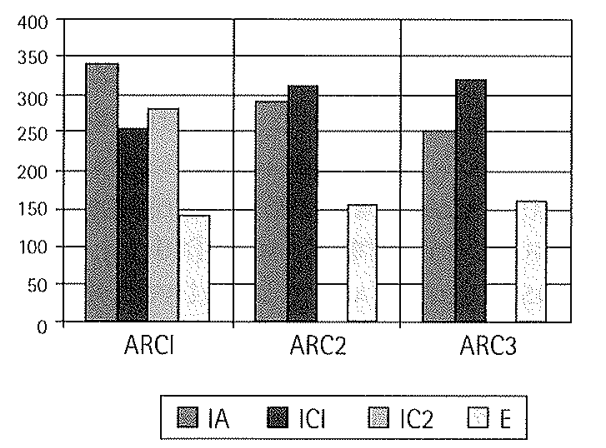

\begin{tabular}{l|l} 
Figura 2 & $\begin{array}{l}\text { Indice de participación conversacional en } \\
\text { las tres grabaciones (turnos). }\end{array}$
\end{tabular}

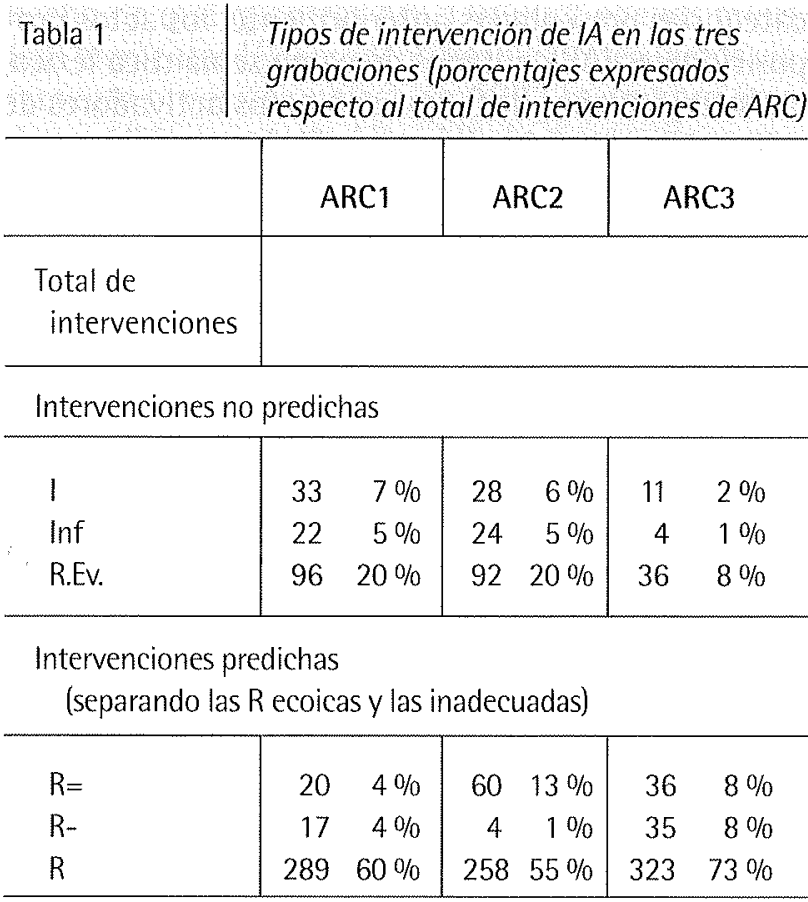

emisiones emitidas por ARC en esa grabación. Como vemos, las grabaciones ARC1 y ARC2 muestran un interesante porcentaje de turnos que ARC emite por iniciativa propia, sin esperar a que nadie le ceda la palabra explicitamente (en total, un $32 \%$ en ARC1 y un $31 \%$ en ARC2); este tipo de emisiones no predichas, sin embargo, desciende drásticamente en ARC3 hasta un $11 \%$.

\section{Actos de habla}

El paso siguiente en el análisis consiste en la clasificación de los actos de habla incluidos en las intervenciones. La tabla 2 muestra la presencia de las

Tabla 2

Número de actos de habla de lA en las tres grabaciones

\begin{tabular}{l|r|r|r}
\hline & ARC1 & ARC2 & ARC3 \\
\hline AP & 83 & 134 & 193 \\
Int & 0 & 26 & 26 \\
AL & 242 & 144 & 44 \\
AB & 30 & 69 & 42 \\
PO & 4 & 2 & 0 \\
PV & 19 & 6 & 4 \\
PC & 0 & 0 & 0 \\
NV & 189 & 291 & 161 \\
Total actos & 567 & 672 & 470 \\
\hline
\end{tabular}


categorías presentadas anteriormente: los actos proposicionales (AP), dotados de carga semántica léxica; las interjecciones (Int), que son actos activadores de inferencias; los actos locutivos (AL), cuya semanticidad es meramente locutiva y no tiene carga léxica; las tareas de edición, que incluyen pausas oralizadas (PO), pausas vacias (PV) y preguntas confirmatorias (PC) y, por último, los actos no verbales (NV) considerados pertinentes en la transcripción.

\section{Longitud media de turno}

La tabla indica los datos concretos referidos a la longitud media de turno en este paciente. Como puede apreciarse, la tercera grabación muestra claramente la recuperación léxica y el descenso interactivo, pues el incremento léxico (junto a la frecuencia de iteraciones) es paralelo a un descenso en efectividad ilocutiva.

\section{Pragmática textual}

Los datos, de estas tres grabaciones apenas ofrecen muestras que permitan un análisis de elementos textuales, por lo que no podemos desarrollar este apartado.

\section{Discusión}

Los datos obtenidos a partir del análisis pragmático de las tres transcripciones nos permiten prestar atención al tipo de turnos que construye el participante afásico, y a la evolución de su gestión comunicativa. Como hemos visto, el nivel de participación de ARC en las tres grabaciones ofrece niveles distintos según tengamos en cuenta su uso de la toma de turno o la cantidad de palabras identificables que logra articular. Desde los planteamientos pragmáticos que asumimos en el presente trabajo, resulta destacable la diferencia cuantita-

Tabla 3

Longitud media de los turnos de ARC en palabras y en actos de habla

\begin{tabular}{l|c|c}
\hline & LMT palabras & LMT actos \\
\hline ARC1 & 2,27 & 1,6 \\
ARC2 & 2,7 & 2,3 \\
ARC3 & 3,3 & 1,8 \\
\hline
\end{tabular}

tiva según recurramos a uno u otro factor de medida: las figuras 1 y 2 muestran que, expresada en turnos, la participación conversacional del paciente afásico está equilibrada con la del resto de participantes (no olvidemos que el objetivo asumido por todos para la grabación es precisamente tal participación); por el contrario, cuando limitamos la cuantificación a elementos del lexicón (incluyendo también seudopalabras), esa participación se reduce drásticamente en las tres grabaciones, con el predominio claro del interlocutor-clave. Tales diferencias podrian constituir un primer argumento de partida para defender la preservación pragmática en la afasia.

En el nivel de la orientación interactiva de los turnos encontramos otro elemento destacable: la evolución desde un estadio en que el hablante afásico presenta una notable iniciativa conversacional, con la elaboración de turnos desencadenantes (inicios) y en general no predichos (informes, reaccio-nes evaluativas), hacia un estadio (ARC3) en que disminuyen llamativamente tales intervenciones. Este predominio de turnos reactivos predichos en ARC3 $(89 \%)$ podría ser interpretado, en parte, como un sintoma del "acoplamiento discursivo" que se produce entre el hablante afásico y su interlocutor-clave, de tal manera que el primero pasa a apoyarse mucho más en la gestión dialógica del segundo (Ferguson, 2000). También podría aducirse que influye el número de interlocutores, pues al reducirse aumenta la eficiencia de las relaciones de predictibilidad entre turnos, pero el peso de este factor es sólo relativo, pues aunque en ARC 1 hay dos interlocutores-clave (esposa e hija, con apenas un par de intervenciones del nieto), en ARC2 la situación es la misma que en ARC3 (o sea, un solo IC) y los actos predichos de ARC se sitúan en el mismo nivel que en ARC1 (en concreto, un $69 \%$ ).

Por lo que se refiere a los actos de habla concretos que rellenan las intervenciones del paciente, y frente a otras grabaciones del corpus PerlA en que es más fácil identificar las palabras emitidas por el interlocutor afásico, en este caso (especialmente en ARC1 y ARC2) apenas es posible aislar palabras concretas; casi todas las emisiones del participante afásico parecen responder a la categoría que hemos llamado "acto locutivo", pues proporcionan un soporte vocal pero casi nunca verbal al acto ilocutivo cuya efectividad transmiten; lo Ilamativo, sin embargo, es que esos turnos aparentemente indescifrables cumplen su papel en el encadenamiento conversacional. De ahi la dificultad añadida para intentar discriminar entre actos locutivos (sonidos carentes de valor proposicional) y lo 
que podrian ser pausas oralizadas (sonidos de relleno que tratan de mantener el turno de habla). Obviamente, esta clasificación funcional podría completarse con un análisis que atendiera sólo a la fuerza ilocucionaria/de cada acto, por ejemplo a partir de la distinción clásica de Searle de actos representativos, expresivos, directivos, proposicionales y declarativos.

También nos hemos encontrado problemas al tratar de reconocer la orientación ilocutiva que el paciente imprime a sus turnos; según uno de los principios básicos del análisis conversacional que considera todo turno cómo depositario de un análisis implicito de los turnos previos (Stubbs, 1983) y habida cuenta de que en la mayoría de los casos esa intención se vehicula con actos locutivos o con interjecciones, dependemos tan sólo de los turnos inmediatos para saber cómo ha sido interpretada por los interlocutores. Por ejemplo, el turno 0219 en el siguiente fragmento de ARC1 ( $\mathrm{H}$ : hija; $\mathrm{M}$ : esposa; I: informante afásico):

$0216 \mathrm{H}$ : a ver/ ya empezamos $\downarrow$ como todas las tardes// a ver qué quieres que te diga $\rightarrow$ a veeer/ yo le digo $\uparrow$ te digoo/que le digas a Beatriz $\rightarrow$ que tienes una perrita $\rightarrow / /$ que se Ilama [Luna]

$0217 \mathrm{M}$ : [Luna]

$0218 \mathrm{H}:$ y no la podemos tocaar $\uparrow$ ni pegar $\uparrow$ ni nada $\uparrow$

0219 I: aah (SIGUE CABIZBAJO, LA CARA APOYADA EN EL PUÑo)

$0220 \mathrm{H}$ : porque te enfadas $\uparrow$ sólo quieres que estar con ella $\uparrow$

Hemos de precisar que la orientación interactiva de los turnos ha sido calculada tanto para las emisiones verbales como para las que son exclusivamente no verbales, por ejemplo, el turno 0247 del siguiente extracto es obviamente un turno que funciona como Respuesta [R]:

$0240 \mathrm{M}:$ mañana

0241 l: ta jool $\uparrow$ (SE SEÑALA A SI MISMO ${ }^{R}$ )

$0242 \mathrm{M}$ : túl y yoo $\uparrow$ al Clínicoo $\uparrow$

0243 l: aah eih

$0244 \mathrm{M}$ : a- al logopeda

0245 I: *(AL LOGOPEDA)*

$0246 \mathrm{M}$ : al logopeda

$0247 \mathrm{l}:$ (ASENTIMIENTO)

Si atendemos a la evolución de las tres grabaciones el primer elemento que destaca es, obviamente, el hecho de que mientras ARC1 y ARC2 apenas muestran elementos léxicos identificables (o sea, actos claramente proposicionales), en ARC3 la proporción es de un $50 \%$ (suma de $A B+A P$ ). La figura 3 nos permite observar dos evoluciones claras en las tres grabaciones: paralelamente al incremento de actos proposicionales observamos una disminución de los actos locutivos, lo que supone que la recuperación gramatical ha evolucionado con independencia de la eficacia pragmática.

En ARC3 encontramos un elemento más que llama la atención y que en ARC2 se apuntaba ya levemente, y es la construcción repetida de las emisiones; no sólo cobran fuerza los actos proposicionales, esto es, las palabras identificables ya como entidades de la lengua, sino que su emisión se convierte en una repetición rápida del elemento léxico en cuestión; estas iteraciones parecen ser incontrolables por el participante afásico, y en ocasiones se producen con un claro aceleramiento articulatorio (figs. 4 y 5 ).

Este tipo de emisiones "en ráfaga" nos hace pensar en la caracterización de la afasia como una alteración de los procesos de inhibición verbal propios de las lenguas naturales. Estas verbalizaciones evidencian una dificultad para silenciar los procesos mentales de búsqueda de la palabra, y pueden ser consideradas una huella de lo que Hernández-Sacristán (2006) ha identificado como un déficit en la capacidad inhibitoria; según este autor, la capacidad estratégica de inhibir la exteriorización fónica del lenguaje aparece alterada en los sujetos con afasia, de tal manera que los signos lingüisticos pasan del estatuto de símbolo (signo del que cabe realizar un uso estratégico) al estatuto de señal.

Por lo que se refiere a la longitud media de turno, cabe señalar su pertinencia en el análisis de la fluidez de cierto paciente. La fluidez puede abordarse desde un

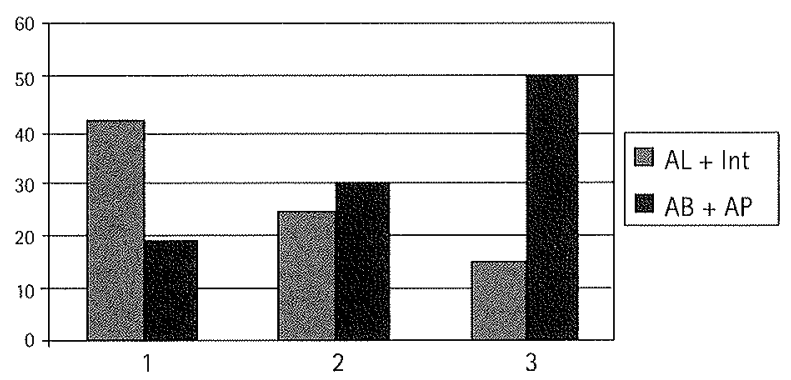

Figura 3 Evolución inversa d actos proposicionales y actos locutivos en las tres grabaciones (en porcentajes), 


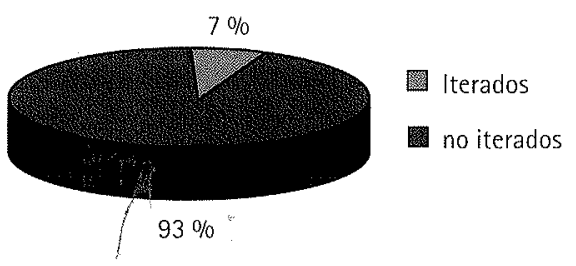

\begin{tabular}{l|l} 
Figura 4 & $\begin{array}{l}\text { Iteración de actos proposicionales en } \\
A R C 2 .\end{array}$
\end{tabular}
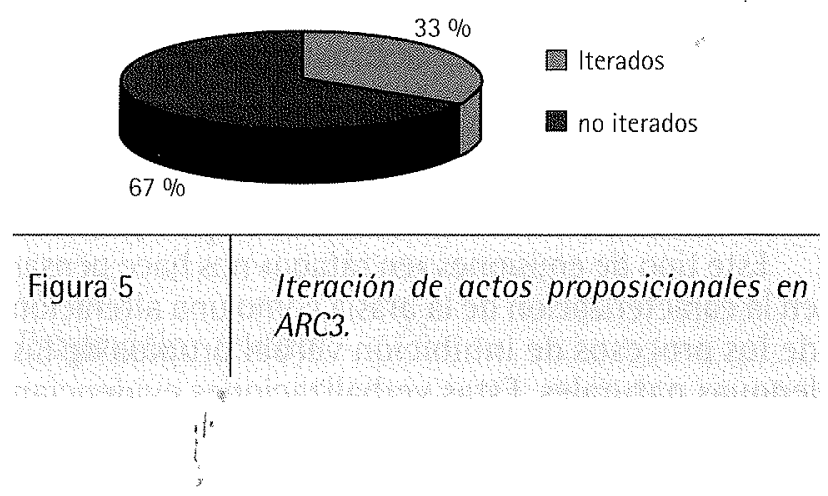

enfoque cuantitativo (número de unidades), pero también cualitativo, buscando construcciones acordes al orden SVO y a los patrones sintácticos propios del código oral; por el contrario, la fluidez ilocutiva se analiza en términos de predictibilidad (Fernández, Rios, Santos, Casadevall, Tejero, López Garcia y cols., 2002).

En definitiva, la integración de una metodologia cuantitativa básica con el análisis cualitativo propio de la pragmática nos conduce a establecer mecanismos de descripción objetivables, asi como a identificar las categorías pragmáticas concretas que resultan más o menos deficitarias en cada sujeto. Surge así un modelo de evaluación pragmática que nos ha permitido dar cuenta de la evaluación de un mismo hablante en tres momentos de su evolución. Tal análisis nos lleva a una conclusión fundamental: la descompensación entre los aspectos gramaticales y pragmáticos en la evolución de ARC. Es necesario, pues, que junto a la práctica rehabilitadora habitual, el diseño de programas de intervención logopédica considere como elemento indispensable la potenciación y el mantenimiento de aquellas conductas que, sin responder exactamente a usos gramaticales normativos, contribuyen a desarrollar la eficacia comunicativa.

\begin{tabular}{|c|c|c|c|c|c|}
\hline Test de Boston & & \multicolumn{2}{|c|}{ Febrero 2004} & \multicolumn{2}{|c|}{ Enero 2006} \\
\hline \multirow[t]{2}{*}{ Escala de severidad } & & \multicolumn{2}{|c|}{$1 / 5$} & \multicolumn{2}{|c|}{$1 / 5$} \\
\hline & & P.D. Paciente & P.D. Muestra & P.D. Paciente & P.D. Muestra \\
\hline \multirow[t]{5}{*}{ Fluidez } & Agilidad articulatoria & \multicolumn{2}{|c|}{ Defectuosa } & \multicolumn{2}{|c|}{ Defectuosa } \\
\hline & Longitud de la frase & 2 & 7 & 2 & 7 \\
\hline & Forma gramatical & \multicolumn{2}{|c|}{ Alterada } & \multicolumn{2}{|c|}{ Alterada } \\
\hline & Parafasia & \multicolumn{2}{|c|}{ No valorable } & \multicolumn{2}{|c|}{ Fonética } \\
\hline & Encontrar palabras & \multicolumn{2}{|c|}{ Proporcional a fluidez } & \multicolumn{2}{|c|}{ Proporcional a fluidez } \\
\hline \multirow[t]{4}{*}{ Comprensión auditiva } & Discriminación palabras & 8 & 72 & 55 & 72 \\
\hline & Identificación partes del cuerpo & 5 & 20 & 11 & 20 \\
\hline & Órdenes & 4 & 15 & 15 & 15 \\
\hline & Material ideativo complejo & 1 & 12 & 5 & 12 \\
\hline \multirow[t]{5}{*}{ Expresión oral } & Agilidad oral -no verbal & 3 & 12 & 6 & 12 \\
\hline & -verbal & 4 & 14 & 9 & 14 \\
\hline & Secuencias automatizadas & 4 & 8 & 6 & 8 \\
\hline & Recitado & 0 & 2 & 0 & 2 \\
\hline & Canto & 1 & 2 & 2 & 2 \\
\hline
\end{tabular}




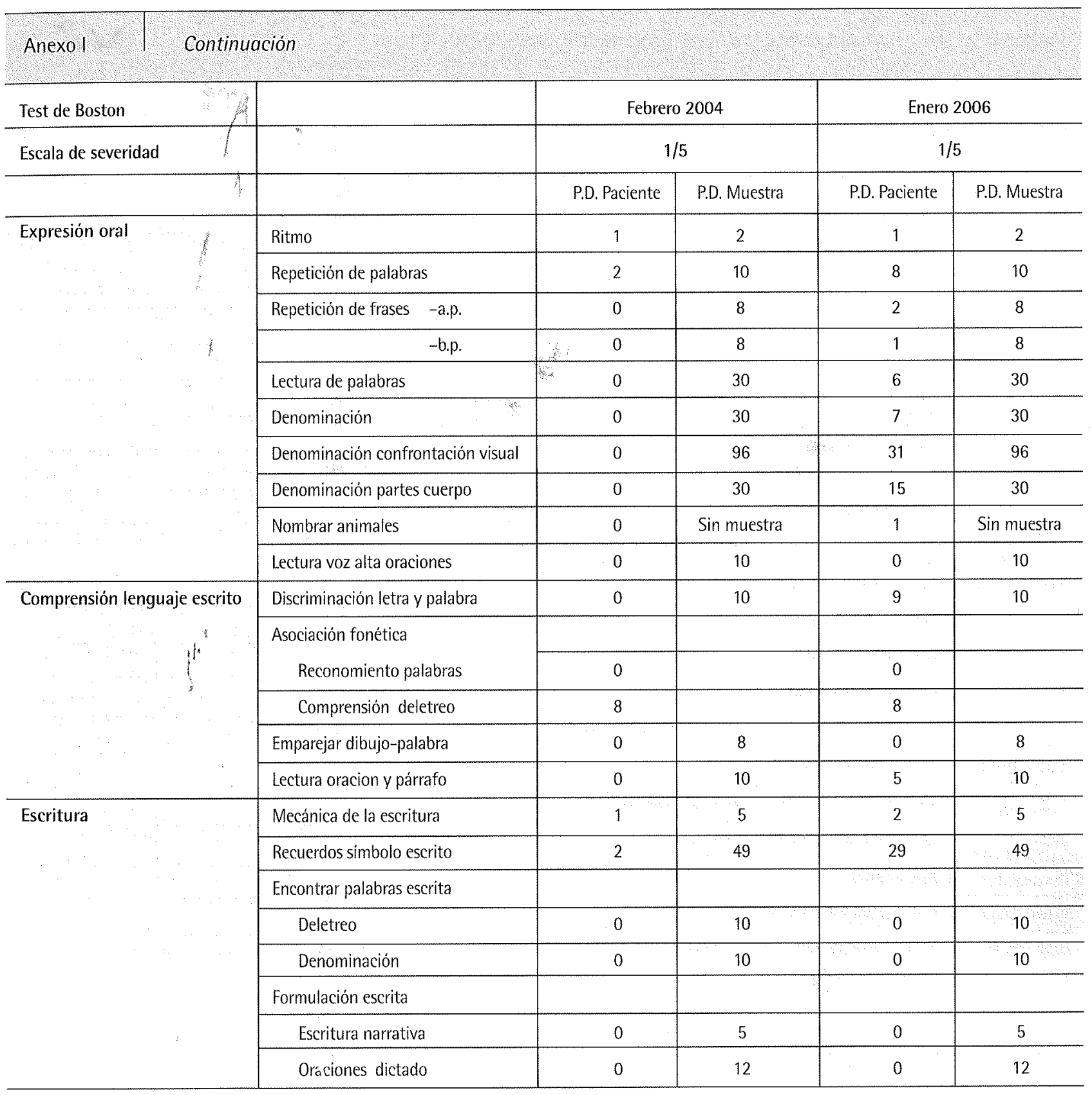

Informe de evaluación logopédica de ARC en enero de 2004. En la exploración anatómica no se observan alteraciones de interés. La agilidad articulatoria es lenta. Presenta un habla no fluente con empleo de expresiones incompletas, ccolálicas e inacabadas. Ausencia de palabras funcionales y numerosos intentos de acceso léxico; apoyo continuo en onomatopeyas y gestualidad. Lenguaje con automatismos alterados con necesidad de inferencias. Denominación severamente afectada (Boston $7 / 30)$ que mejora al facilitar claves fonéticas. Repetición preservada para palabras sencillas y alterada para palabras complejas y frases cometiendo parafasias fonéticas, omisiones y distorsiones neológicas. Comprensión oral con problemas similares a los de la sintomatología expresiva; (TOKEN TEST 6/36); comprensión conservada para uso conversacional, muy dependiente del contexto. Lectura alterada tanto en palabras como en frases, alteración del proceso de conversión grafemamfonema. Escritura con agrafía afásica.

Informe de evaluación logopédica de $A R C$ en enero de 2006. Habla no fluente, disprosódica, agramática, perseverativa y taquilálica. Empleo de expresiones y formas sobreaprendidas; ausencia de palabras funcionales y numerosos intentos de acceso léxico; facilita la comprensión al interlocutor mediante onomatopeyas y gestualidad. Lenguaje con automatismos conservados, salvo el abecedario. Denominación severamente afectada (Boston 13/30) que mejora al facilitar claves fonéticas. Repetición alterada para palabras complejas y/o compuestas y para oraciones de más de cuatro elementos gramaticales, cometiendo parafasias fonéticas, omisiones y distorsiones neológicas. Repetición de seudopalabras preservada. Comprensión oral con problemas similares a los de la sintomatologia expresiva; deterioro para comprender las oraciones gramaticales complejas (TOKEN TEST 17'5/36); conservada para el uso conversacional, pero muy dependiente del contexto y de los canales no verbales. Lectura global de ciertas palabras de uso común; rendimiento bajo en la asociación dibujo-palabra por la pérdida de la lectura. Lectura en voz alta alterada tanto en palabras como en frases, alteración del proceso de conversión grafema-fonema. Escritura con agrafia afásica; grafia de trazo seguro, forma redondeada, buena prensión y regularidad normalizada; mecánica de la escritura preservada salvo el abecedario. Dictado de letras y dígitos conservado, pero dictado de palabras alterado, con paragrafias y neologismos. Expresión escrita severamente afectada. 


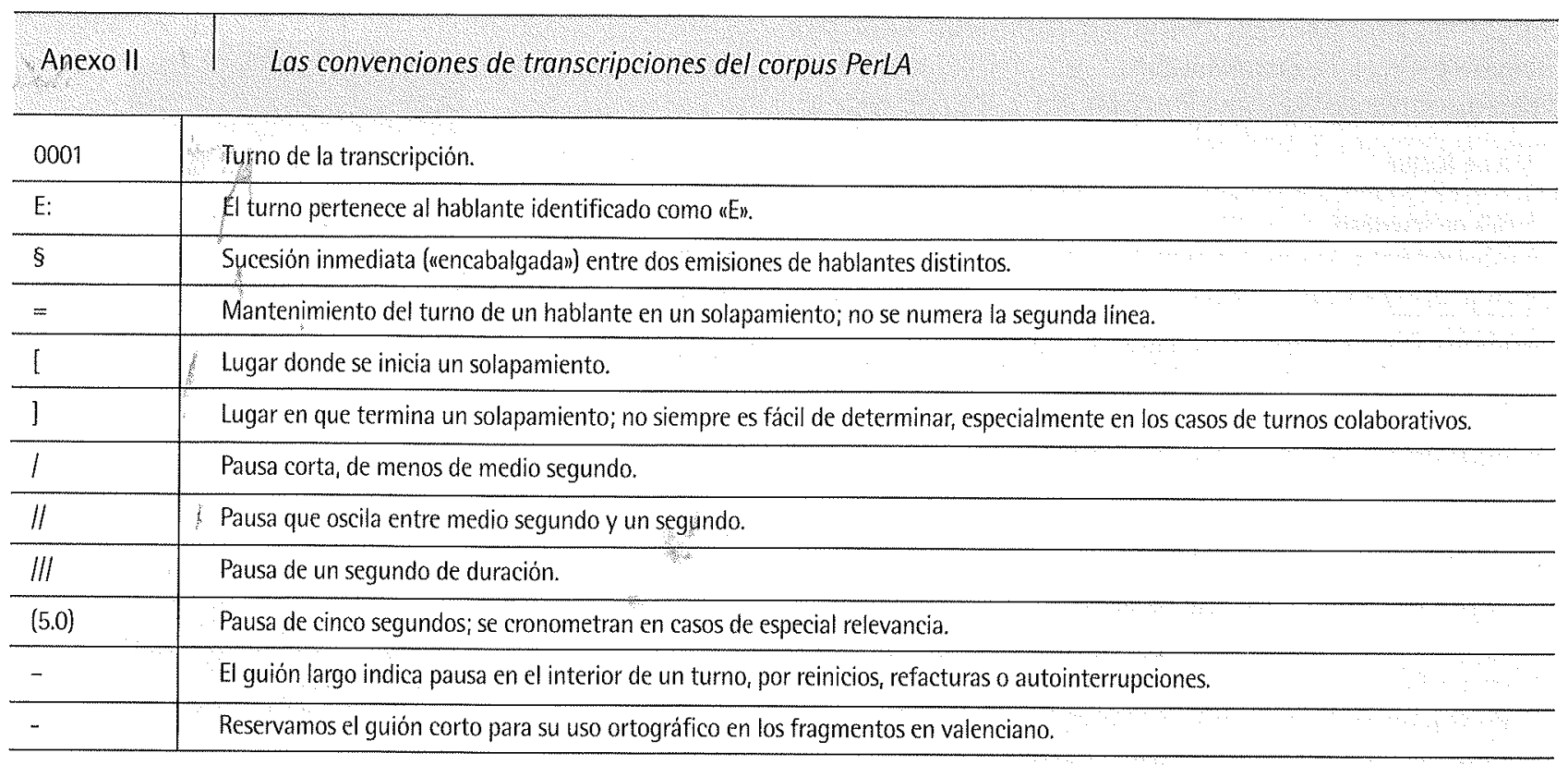

MARCAS TONALES: se indican las entonaciones que no coinciden con los patrones melódicos habituales o que tengan función demarcativa en interior de intervención. Toda marca tonal supone la existencia de una minima pausa, por lo que no resulta necesario consignar la pausa breve (l) cuando se marca la entonación.

\begin{tabular}{|c|c|}
\hline$\rightarrow$ & Entonación suspendida. \\
\hline $1 \quad t^{3}$ & Entonación ascendente. \\
\hline 3 & Entonación descendente. \\
\hline${ }^{\circ}()^{\circ}$ & Pronunciación en voz muy baja, próxima al susurro; a veces son emisiones de uhablar para uno mismon. \\
\hline MAYÚSCULA & Pronunciación en voz muy alta. \\
\hline$*()^{*}$ & Pronunciación extraña: entrecortada, costosa o alterada. \\
\hline ple)ro & Reconstrucción de un fragmento que no ha sido pronunciado por el hablante. \\
\hline$h$ & Aspiraciones. \\
\hline m'han dicho & Elisiones por fonética sintáctica y velocidad de pronunciación. \\
\hline $\begin{array}{l}\text { di ga me/lo } \\
\text { que ve/ en la } \\
\text { fo to }\end{array}$ & $\begin{array}{l}\text { La negrita se utiliza para marcar una pronunciación "silabeadan, que el hablante realiza con especial cuidado; puede darse en una pala- } \\
\text { bra o en una frase. }\end{array}$ \\
\hline cursiva & Fragmentos de estilo directo o metalenguaje. \\
\hline$(x x x \quad x x x)$ & Fragmento indescifrable, aparentemente de dos palabras. \\
\hline ((la doctora) $)$ & Transcripción dudosa; el transcriptor propone una posibilidad pero no está seguro. \\
\hline $\begin{array}{l}\text { aqui } \downarrow \text { / } \\
\text { (SE TOCA LA } \\
\text { FRENTE) }\end{array}$ & $\begin{array}{l}\text { Gestos y elementos no verbales que aclaran la intervención. Algunos de estos gestos aparecen con su nombre concreto: emblemas } \\
\text { como el EMBLEMA DEL BESO, reguladores como el DE COMPÁS o ilustradores como el BARRIDO O el INTENSIFICADOR. }\end{array}$ \\
\hline aquí $\left(\operatorname{RISAS}^{\mathrm{i}}\right)$ & La $R$ en superíndice significa que el gesto en cuestión se realiza repetidamente. \\
\hline$\Leftrightarrow \mathrm{E})$ & El hablante dirige la mirada hacia $E$. \\
\hline$\prod_{1} \|_{1} \Leftrightarrow$ & Dirección en que se realiza cierto movimiento, por ejemplo (BRAzo $\downarrow)$ \\
\hline
\end{tabular}




\section{Bibliografía}

Adams, C. (2002). Practicioner review: The assessment of language pragmatics. Journal of Child Psychology and Psychiatry, 43(8), 973-987.

Adams, Catherine y Bishop, Dorothy V. (1989). Conversational characteristics of children with semantic-pragmatic disorder. 1: Exchange structure, turntaking, repairs and cohesion. British Journal of Disorders of Communication, 24, 211-239.

Ahlsén, E. (1995). Pragmatics and Aphasia. An Activity Based Approach. Gothenburg Papers in Theoretical Linguistics, 77

Allerton D. J. (1969). The sentence as a linguistic unit. Lingua, 22, 27-46

Austin, J. L. (1962). How to do things with words. Oxford: University Press.

Baixauli-Fortea, I., Roselló, B. y Miranda-Casas, A. (2004): Evaluación de las dificultades pragmáticas. Estudio de casos, Rev Neurol, 38(Supl. 1), 69-79.

Brownell, H. y Stringfellow, A. (1999). Making Requests: Illustrations of How Right-Hemisphere Brain damage Can Affect Discourse Production. Brain and Language, $68(3), 442-465$

Bruner, J. S. (1993). Explaining and Interpreting: Two Ways of Using Mind. En G. Harman (Eds.), Conceptions of the human mind: essays in honor of George A. Miller (pp. 123-137). Hillsdale, $\mathrm{NJ}$ : Lawrence Erlbaum.

Bryan, K. L. (1989). Thd Right Hemisphere Language Battery. London: Whurr Pub.

Champagne, M., Dešautels, M.C. y Joanette, Y. (2003). Accounting for the pragmatic deficit in RHD individuals: A multiple case study. Brain Lang, 87, 210-211.

Conti-Ramsdem, G. y McTear, M. (1989). Assessment of Pragmatics. En Grundy, K. (Ed.). Linguistics in clinical practice. London: Whurr Publ.

Creaghead N. (1984). Strategies for evaluating and targeting pragmatics behaviours in young children. Seminars in Speech and Lanquage, 5, 241-252.

Crockford, C. y Lesser, R. (1994). Assessing functional communication in aphasia: Clinical utility and time demands of three methods. European Journal of Disorders of Communication, 29, 165-182.

Davidson, J. (1984). Subsequent versions of invitations, offers, requests and proposals dealing with potential or actual rejection. En Atkinson, J.M. y Heritage, J. (Eds.) Structures of Social Action (pp. 102-128). Cambridge: University Press.

Davis, G. A y Coelho, C. A. (2004). Referential cohesion and logical coherence of narration after closed head injury. Brain Lang, 89, 508-523.

Dewart, H. y Summers, S. (1995). Pragmatics Profile of Everyday Communication Skills in Children. Windsor: Nfer-Nelson.

Dewart, H. y Summers, S. (1996). Pragmatics Profile of Everyday Communication Skills in Adults. Windsor: Nfer-Nelson.

Dijkstra, K., Bourgeois, M. S., Allen, R. S. y Burgio, L. D. (2004). Conversational coherence: discourse analysis of older adults with and without dementia. Joumal of Neurolinguistics, 17, 263-283.

Dubois, B. y Crouch, I. (1975). The question of tag questions in women's speech: they don't really use more of them. do they? Language in Society, 4, 289-294.
Eckman, P. y Friesen, W. V. (1969). The repertoire of nonverbal behavior: categories, origins, usage and codings. Semiotica, 6, 238-252.

Ferguson, A. (2000). Maximising Communication Effectiveness. En Müller, N. (Ed.), Pragmatics in speech and language pathology. Studies in clinical applications (pp. 53-88). Amsterdam/Philadeplphia: John Benjamins.

Fernández, T., Ríos, C., Santos, S., Casadevall, T., Tejero, C., López-García, E., y cols. (2002). 'Cosas en una casa', una tarea alternativa a 'animales' en la exploración de la fluidez verbal semántica: estudio de validación. Rev Neurol, 36(6), 520-523.

Fishman, J. A. (1965). Who speaks what language to whom and when? Linguistique, 2, 67-88.

Gallardo-Paúls B. (2005). Afasia y conversación. Las habilidades comunicativas del interlocutor-clave. Valencia: Tirant lo Blanch.

Gallardo-Paúls, B. (1993). La transición entre turnos conversacionales: silencios, interrupciones y solapamientos. Contextos, XI(21-22), 189-220.

Gallardo-Paúls, B. (1993). Lingüistica perceptiva y conversación: secuencias. Valencia: Universitat.

Gallardo-Paúls, B. (2002). La investigación de los déficits pragmáticos. En Luque, J. D., Pamies, A. y Manjón, F. (Eds.). Nuevas tendencias en la investigación lingüistica (pp. 525-538). Granada: Método Ediciones.

Gallardo-Paúls, B. (2005) Categorias inferenciales en pragmática clínica. Rev Neurol, 41(Supl. 1),65-71.

Gallardo-Paúls, B. y Sanmartín, J. (2004). Propuesta de transcripción de datos afásicos. VI Congreso de Lingüistica General, 3-7 de mayo de 2004. Santiago de Compostela.

Gallardo-Paúls, B. y Marin-Jordá, M. J. (2005). Marcadores discursivos procedentes de verbos de percepción en el discurso afásico. Revista de Investigación Lingüistica, 3 , 53-94.

Gallardo-Paúls, B. y Moreno-Campos, V. (2005a). Afasia fluente. Materiales y análisis pragmático (Volumen 2 de/ corpus PerLA). Valencia: Universitat/AVaLCC

Gallardo-Paúls, B. y Moreno-Campos, V. (2005b). Estrategias colaborativas de compensación del décifit lingüistico: la importancia del interlocutor-clave en el indice de participación conversacional. Pragmalingüistica, 13, 87-113.

Gallardo-Paúls B. y Hernández-Sacristán, C. (2006). Anotaciones a un texto conversacional: la Agilidad del Turno y el Índice de Participación Conversacional en la afasia. Lynx. Annexa, 14, 39-58.

Gallardo-Paúls, B. y Sanmartín J. (2005). Afasia no fluente. Materiales para su estudio. (Volumen 1 del corpus Per(A). Valencia: Universitat/AVaLCC.

Gardner, H. y Brownell, H.H. (1986). Right Hemisphere Communication Battery, Boston Psychology Service.

Gerber, S. y Gurland, G.B. (1989). Applied pragmatics in the assessment of aphasia. Seminars in Speech and Language, 10, 263-281.

Grice, H. P. (1957). Meaning. Philosophical Review, 66, 377-88.

Hamel, C., Giroux, F. y Joanette, Y. (2003). Inferential abilities in right-hemisphere-damaged individuals: Looking for subgroups. Brain Lang, 87, 206-07.

Hays, S. J., Niven, B. E., Gidfrey, H. P. D. y Linscott, R. J. (2004). Clinical Assessment of Pragmatic Langua- 
ge Impairment: A generalisability study of older people with Alzheimer disease. Aphasiology, 18(8), 693 714.

Hernández-Sacristán, C. (2006). Inhibición y lenguaje. A propósito de la afasia y la experiencia del decir. Madrid: Biblioteca Nueva.

Hillert, D. G. (2004). Spared access to idiomatic and literal meanings: a single-case approach. Brain Lang, 89, 207215.

Huber-Okrainec, J., Blaser, S.E. y Dennis, M. (2005). Idiom comprehension deficits in relation to corpus callosum agenesis and hypoplasia in children with spina bifida meningomyelocele. Brain Lang, 93(3), 34968.

Huber-Okrasinec, J. y Dennis, M. (2003). Idiom comprehension in chilhood: an assessment tool and age norms. Brain Lang, 87, 188-191.

Jaffe, J. y Feldstein, S. (1970). Rythms of Dialogue. New York: Academic Press.

Jefferson, G. (1989). Preliminary Notes on a Possible Metric which Provides for a Standard Maximum Silence of Approximately One Second in Conversation. En Roger, D. y Bull, P. (Eds.). Conversation: an interdisciplinary perspective (pp. 166-196). Clevedon: Multilingual Matters.

Jefferson, G. (1992). Sacks' Lectures on conversation. Oxford: Basil Blackwell.

Joanette, Y. y Ansaldo, A. I. (1999). Clinical Note: Acquired Pragmatic,/mpairments and Aphasia. Brain Lang 68, 3 529-534.

Kasher, A., Batori, G., Soroker, N., Graves, D. y Zaidel, E. (1999). Effects on Right- and Left-Hemisfere Damage on Uniderstanding Conversational Implicatures. Brain Lang, 68(3), 566-590.

Kerbel, D. y Grunwell, P. (1998). A study of idiom comprehension in children with semantic-pragmatic difficulties, II: Between-groups results and discussion. International Journal of Language and Communication Disorders, $33(1), 23-44$.

Labos, E., Zabala, K., Atlasovich, C., Pavón, H. y Ferreiro, J. (2003). Evaluación de funciones lingüistica y comunicativas en pacientes con lesión de hemisferio derecho. Revista Neurológica Argentina, 28, 96-103

Labos, E., Del Rio, M., Zabala, K., y Nespoulos, J. L. (2005). Protocolo Montréal-Toulouse-Buenos Aires de examen lingüistico de la afasia, Buenos Aires: Eds. Lenguaje y Congnición.

Labov, W. (1972). Modelos sociolingüisticos. Madrid: Cátedra, 1983.

Linscott, R. J., Knight, R. G., Godfrey, H. (1996). The profile of functional impairment in communication (PFIC): a measure of communication impairment for clinical use. Brain Injury, 10, 397-412.

López-Garcia, A. (1989). Fundamentos de lingüística perceptiva. Madrid: Gredos.

Marini, A., Carlomagno, S., Caltagirone, C. y Nocentini, U. (2005). The role played by the right hemisphere in the organization of complex textual structures. Brain Lang, 93, 46-54.

Moreno-Campos, V. (2006). Afasia y tempo dialógico: el indice de participación conversacional. En Gallardo, B. Hernández, C. y Moreno, V. (Eds.). Lingüistica clínica y neuropsicología cognitiva. Actas del Primer Congreso
Nacional de Lingüistica Clínica. Vol. 1: Investigación e intervención en patologias del lenguaje. Valencia: Universitat, 87-16.

Muñoz-Céspedes, J.M. y Melle, N. (1999). Alteraciones de la pragmática de la comunicación después de un traumatismo craneoencefálico. Rev Neurol, 38 (9), 852859.

Nenonen, M., Niemi, J. y Laine, M. (2002). Representation and processing of idioms: evidence from aphasia. Journal of Neurolinguistics, 75, 43-58.

Papagno, C., Tabossi, P., Colombo, M. R. y Zampetti, P. (2004). Idiom comprehension in aphasic patients. Brain Lang, 89, 226-234.

Papagno, C. y Genoni, A. (2003). The role of syntactic processing idiom comprehension. Brain Lang, 87, 73-74.

Penn, C. (1985). The Profile of Communicative Appropriateness: A clinical tool for the assessment of pragmatics. The South African Journal of Communication Disorders, $32,18-23$

Penn, C. (2000). Clinical Pragmatics and Assessment of Adult Language Disorders. Process and Product. En Müller, N. (Ed.). Pragmatics in speech and language pathology. Studies in clinical applications (pp. 107-124). Amsterdam/Philadelphia: John Benjamins.

Perkins, L., Withworth, A. y Lesser, R. (1998). Conversing in dementia: A conversation analytic approach. En Paradis M. (Ed.). Pragmatics in Neurogenic Communication Disorders (pp. 33-53). New York: Elsevier Sc.

Phelps-Terasaki, D. y Phelps-Gun, T. (1992). Test of Pragmatic Language. Hove: Psychological Corporation.

Prutting, C. A. y Kirchner, D. M. (1983). Applied pragmatics. En Gallagher, T. M. y Prutting, C. A. (Eds.). Pragmatic assessment and intervention issues in language (pp. 29-64). San Diego, California: College-Hill Press.

Prutting, C. A. y Kirchner, D. M. (1987). A clinical appraisal of the pragmatic aspects of language. Journal of Speech and Hearing Disorders, 52, 105-119.

Puyuelo, M., Wiig, E. H., Renom, J. y Solanas. (1997). BLOC. Bateria de Lenguaje Objetiva y Criterial. Barcelona: Masson.

Ripich, D. N., Carpenter, B. D. y Ziol, E. (2000). Conversational cohesion patterns in men and women with Alzheimer's Disease: a longitudinal study. International Journal of Language and Communication Disorders, 35 49-64.

Sacks, H., Schegloff, E. y Jefferson, G. (1974). A symplest systematics for the organization of turn-taking for conversation. Language, 50 (4), 696-735.

Schmidt, G. L., DeBuse, C. J. y Seger, C. A. (2005). Right Hemisphere metaphor processing? Characterizing the lateralization of semantic processes. Broin Lang i.p.

Schulman, B. B. (1985). Test of Pragmatics Skills. Tueson, Ar: Communication Skill Builders.

Searle, J. (1976). A classification of illocutionary acts. Language in society, 5, 1-23.

Stubbs, M. (1983). Análisis del discurso. Madrid: Alianza.

Tartter, V. C., Gomes, H., Dubrovsky, B., Molholm, S. y Stewart, R. V. (2002). Novel Metaphors Appear Anomalous at Least Momentarily: Evidence from N400. Brain Lang, 80, 488-509. 
Terrell, B.Y. y Ripich, D.N. (1989). Discourse competence as a variable in intervention. Seminars in Speech and Language, 10, 282-297.

Tomoeda, C. K. y Bayles, K. A. (1993). Longitudinal effects of Alzheimer's disease on discourse production. Alzheimer Disease and Associated Disorders, 4, 223-236.

Vanhalle, C., Lemieux, S., Ska, B. y Joanette Y. (2000). The Evaluation of the Ability of Right-Hemisphere-Damaged Patients to Process Speech Acts: An Ecological Approach. Brain Lang, 74, 483-486.

Vázquez-Veiga, N. (2003). Marcadores discursivos de recepción. Santiago de Compostela: Universidade.
Whitworth, A., Perkins, L. y Lesser, R. (1997a). Conversational Analysis Profile for People with Aphasia. London: Whurr Publ.

Whitworth, A., Perkins, L. y Lesser, R. (1997b). Conversational Analysis Profile for People with Cognitive Impairment. London: Whurr Publ.

Recibido: 24/05/06

Modificado: 18/09/06

Aceptado: 20/09/06 